\title{
Assessing Inner Ear Volumetric Measurements by Using Three-Dimensional Reconstruction Imaging of High-Resolution Cone-Beam Computed Tomography
}

\author{
Jan Hiller ${ }^{1}$ (D) Nour-Eldin Abdelrehim Nour-Eldin ${ }^{1,2} \cdot$ Tatjana Gruber-Rouh $^{1} \cdot$ Iris Burck ${ }^{1} \cdot$ Marc Harth $^{1} \cdot$ Timo Stöver $^{3}$ • \\ Thomas Vogl ${ }^{1}$ • Nagy Naguib Naeem Naguib ${ }^{4,5}$
}

Accepted: 9 September 2020 / Published online: 18 September 2020

(C) The Author(s) 2020

\begin{abstract}
The aim of the study was to obtain volumetric data of the components of the inner ear using three-dimensional reconstruction of high-resolution cone-beam computed tomography (CBCT) images. Two hundred three $\mathrm{CBCT}$ image series of the temporal bone from 118 anatomically normal patients (55 women and 63 men; mean age: $49.4 \pm 20.4$ years) with different suspected disorders were included in this study. Normative volumetric measurements of the inner ear, the cochlea, the semicircular canals (SSC), and the vestibule were determined using a semi-automated reconstruction method of the Workstation. Volumetric measurements were successfully completed in all 118 patients. Mean inner ear, cochlear, and vestibule volumes were statistically significantly larger in males than in females on both sides $(p<0.001)$. Regarding the semicircular canals, no statistically significant $(p=0.053)$ volume difference was found. The difference between the volumes on both sides was not significant. No correlation between the patient's age and the volume of the compartments was seen $(p>0.05)$. There was no significant difference between mean bony inner ear volumes when the clinical diagnoses were compared $(p>0.05$ for all clinical diagnoses and volumes). Our study concluded that three-dimensional reconstruction and assessment of the volumetric measurements of the inner ear can be obtained using highresolution $\mathrm{CBCT}$ imaging.
\end{abstract}

Keywords Inner ear · Cone-beam computed tomography · Imaging · Three-dimensional · Organ volume $\cdot$ Semi-automated segmentation $\cdot$ Drug concentration

\section{Introduction}

Various pathological disorders of the inner ear are associated with increased or decreased organ volume, for which exact

Jan Hiller

jan_hiller@hotmail.de

1 Department of Diagnostic and Interventional Radiology, University-Hospital Frankfurt, Theodor-Stern-Kai 7, 60590 Frankfurt, Germany

2 Department of Diagnostic and Interventional Radiology, Faculty of Medicine, Cairo University, Cairo, Egypt

3 Department of Otolaryngology, University Hospital Frankfurt, Theodor-Stern-Kai 7, 60590 Frankfurt, Germany

4 AMEOS Hospital in Halberstadt, Radiology Department, Halberstadt, Germany

5 Department of Diagnostic and Interventional Radiology, Faculty of Medicine, Alexandria University, Alexandria, Egypt measurements of this compartment can be of diagnostic and clinical relevance $[1,2]$. Volumetric measurements are challenging due to the extremely small size, and visual inspection of two-dimensional images is not accurate enough to identify all pathologic disorders [3]. In 2014, Teixido et al. stated that volumetric measurements of the inner ear might be much more accurate when using high-resolution cone-beam computed tomography (CBCT) images with a slice thickness of only 0.1 or $0.2 \mathrm{~mm}$ [4]. CBCT represents a powerful diagnostic tool for radiologic examinations of the temporal bone that has increased in popularity [5-9]. Compared with conventional computed tomography (CT), its advantages are a higher spatial resolution of osseous structures and a reduced effective radiation dose due to the fact that only the anatomical region of interest is imaged [5]. The volume can be measured using a variety of methodological approaches such as $\mathrm{CT}$ or magnetic resonance imaging (MRI). There are numerous possibilities of image processing [4, 10-14]. However, no volumetric assessment of the bony parts of the inner ear has been performed 
using the latest CBCT data. MRI is often used for the fluid parts of the inner ear. Our study was performed to assess volumetric measurements of the bony inner ear components using three-dimensional (3D) reconstruction of highresolution CBCT data and to test the correlation of the volume with respect to patient age, sex, and clinical diagnosis.

\section{Methods}

The study was approved by the local ethical committee (Number 284/17). All patients signed an informed consent before the examination. Between January 2014 and June 2017, 203 CBCT image series of the temporal bone of 118 patients who were referred to our university hospital were examined retrospectively. CBCT of the temporal bone on either side was available in 85 patients $(72 \%)$ and of only one side in 33 patients ( 17 of the right and 16 of the left side; $28 \%$ ). The analysis included measurement of the inner ear volume, the cochlea, the semicircular canals (SSC), and the vestibule. The obtained volumes were compared with regard to patients' age, sex, and inner ear clinical diagnosis.

\section{Patient Group}

The examined patient group consisted of 63 men and 55 women (range: 5 to 87 years; mean age: $49.4 \pm 20.4$ years). CBCT was performed for different reasons. The clinical diagnosis was hearing loss $(n=20)$, tinnitus $(n=3)$, vertigo $(n=6)$, to exclude inner ear anomalies $(n=5)$, suspected cholesteatoma ( $n=23)$, suspected otosclerosis $(n=4)$, and surgical planning of cochlear implant or tympanoplasty $(n=57)$. All patients showed a normal configuration of the inner ear. Therefore, no further selection was necessary, and none of the patients had structural abnormalities. Inclusion criteria were a complete data set of the entire temporal bone with complete coverage of the inner ear and its components. Exclusion criteria were incomplete data sets with missing imaging of the entire inner ear, motion artifacts, and metal artifacts (e.g., due to middle ear prosthesis). Patients with cochlear implants were also excluded due to artifacts.

Imaging data were generated by high-resolution CBCT using the ProMax 3D Max ProFace (Planmeca). The following parameters were used: When applying an intensity of a current of $7 \mathrm{~mA}$ and a voltage of $120 \mathrm{kV}$, time for a complete 360 -degree rotation of the c-arm was about $10 \mathrm{~s}$. Factory preset volume rendering was used. The field of view (FOV) was set to a diameter of $100 \mathrm{~mm}$ and a height of $90 \mathrm{~mm}$ for adults. For children under 18 years, FOV was set to a diameter of $85 \mathrm{~mm}$ and a height of $75 \mathrm{~mm}$. The edge length of the cubic voxel was $150 \mu \mathrm{m}$, and the focal spot was $0.5 \mathrm{~mm} \times 0.5 \mathrm{~mm}$. All patients were positioned seating on a chair holding two handles. Before the actual images were taken, a lateral and frontal radiograph was made to confirm the correct position of the FOV (field of view).

\section{Image Processing}

Volumetric assessment was performed using 3D reconstruction on an Advantage Workstation 4.4 (GE Healthcare). All images had been evaluated by two radiologists with a minimum of 3 and 8 years of experience in head and neck imaging in consensus. The volume viewer tool (Version 7.6.29) allows the user to segment the different tissues in two steps. After visually identifying the structures, the inner ear was separated from the bony labyrinth. Structures of no relevance for this study, such as the internal auditory meatus or cranial nerve canals, were cropped out manually at first. For each side, this was repeated in every single CBCT slice of the patients' images. Semi-automated segmentation was performed in a second step. We achieved the best results using a threshold segmentation algorithm with a voxel gray scale range of -200 to +500 [15] as the desired inner ear contained voxels in this range. Anatomically, the vestibule with the horizontal, posterior, and upper SCC and the cochlea without modiolus and cochlear aqueduct were part of the volume measurements. The inner and external auditory canal, Rosenthal's canal, and the vestibular aqueduct and endolymphatic sac were not included. It was not possible to quantify endolymphatic and perilymphatic spaces, as they cannot be separated using CBCT. The same imaging parameters were used in all patients to ensure homogeneity of the results. For the highest possible validity and to minimize some of the bias, reproducibility of the image acquisition should be ensured. The used gray scale does not correspond to the Hounsfield units (HU) of conventional CT, which is a specific disadvantage of most CBCT scanners. Using this fixed threshold, the Volume Viewer automatically cuts out the inner ear from bony surroundings and creates a 3D reconstruction (Fig. 1; Fig. 2). After the complete segmentation process, a tool automatically computed the volume. Each segmentation process required

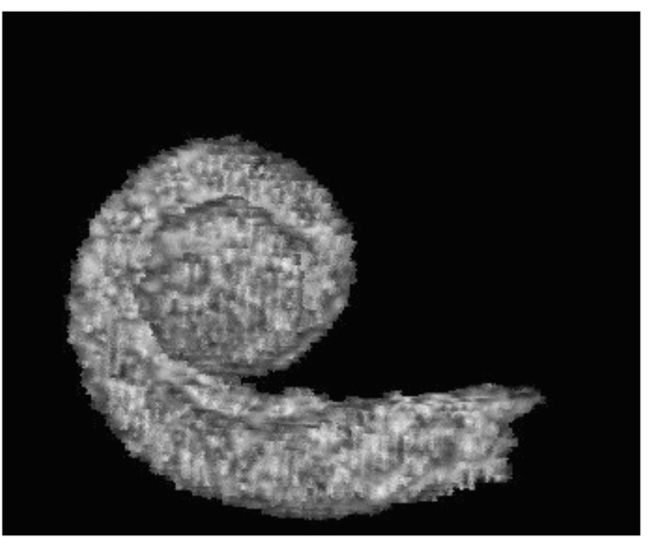

Fig. 1 Three-dimensional reconstruction of the cochlea in a 57-year-old woman presenting with hearing loss. Unsmoothed surface 


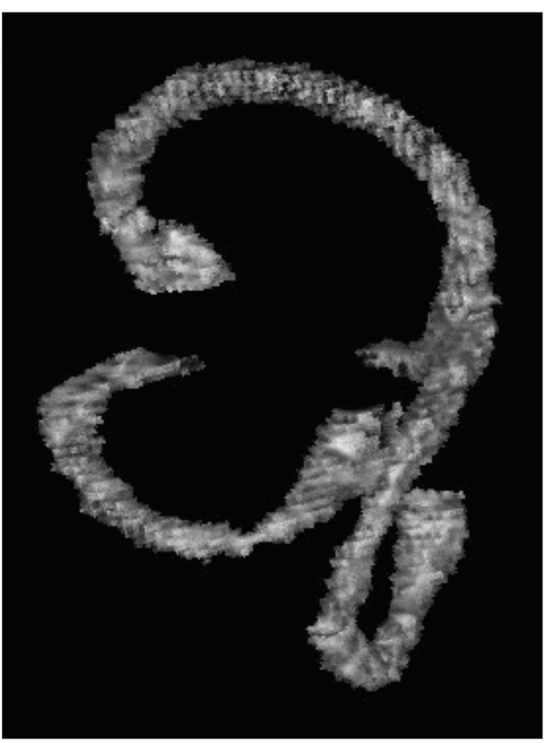

Fig. 2 Three-dimensional reconstruction of the semicircular canals in a 48-year-old woman presenting with hearing loss. Unsmoothed surface

$40 \mathrm{~min}$. All volumetric measurements were reported in cubic millimeters $\left(\mathrm{mm}^{3}\right)$.

\section{Statistical Analysis}

Statistical analysis was performed using a suitable statistics program (BiAS Version 11.09, epsilon Publisher). The results were provided as mean values with the corresponding standard deviations (SD). $p$ values less than 0.05 were considered statistically significant. Pearson's correlation coefficient was determined for the correlation analysis between patient age and inner ear volume. Testing for normal distribution was performed using Kolmogorov-Smirnov-Lilliefors test. As all data was distributed normally, sex-related differences were statistically evaluated with the Students $t$ test. One-way analysis of variance and Scheffe post hoc test were applied to compare mean volumes between different clinical diagnoses.

\section{Results}

$3 \mathrm{D}$ reconstruction and volumetric measurements were successfully completed in all 118 patients (Table 1). Volumes of the inner ear, the cochlea, the semicircular canals, and the vestibule were obtained in all 203 CBCT image series (102 right and 101 left temporal bones).

On the right side, the mean volume of the bony inner ear was $189.5 \mathrm{~mm}^{3}$ (range: $131-267 \mathrm{~mm}^{3}$, SD: $26.3 \mathrm{~mm}^{3}$ ), of the cochlea $78.1 \mathrm{~mm}^{3}$ (range $48-107 \mathrm{~mm}^{3}$; SD $11.5 \mathrm{~mm}^{3}$ ), of the SSC $51.1 \mathrm{~mm}^{3}$ (range $30-89 \mathrm{~mm}^{3}$; SD $11.9 \mathrm{~mm}^{3}$ ), and of the vestibule $59.8 \mathrm{~mm}^{3}$ (range $35-86 \mathrm{~mm}^{3}$, SD $9.5 \mathrm{~mm}^{3}$ ).

On the left side, the mean bony inner ear volume was $193.7 \mathrm{~mm}^{3}$ (range 145-285 $\mathrm{mm}^{3}$; SD $29.1 \mathrm{~mm}^{3}$ ), cochlea volume was $76.4 \mathrm{~mm}^{3}$ (range 53-106 $\mathrm{mm}^{3}$; SD $11.9 \mathrm{~mm}^{3}$ ), SSC volume was $52.6 \mathrm{~mm}^{3}$ (range $33-102 \mathrm{~mm}^{3}$; SD $13 \mathrm{~mm}^{3}$ ), and vestibule volume was $61.9 \mathrm{~mm}^{3}$ (range $40-91 \mathrm{~mm}^{3}$; SD $10.1 \mathrm{~mm}^{3}$ ).

No statistically significant difference in volumes was found when comparing both sides $(p>0.05)$.

No significant correlation between patient age and the volume of the single compartments on the left or right side was seen $(p>0.05)$.

Compared with women, men showed a significantly larger volume of the bony inner ear on the left and right side $(p<0.001$; Fig. 3$)$, of the cochlea on both sides $(p<$ 0.001 ; Fig. 4$)$, and of the vestibule on both sides $(p<0.001$; Fig. 5) (Table 1). Regarding the SCC, no statistically significant volume difference was found when comparing men and women ( $p=0.053$; Fig. 6).
Table 1 Volume results related to gender. Values are expressed as mean \pm standard deviation in $\mathrm{mm}^{3}$

\begin{tabular}{llrr}
\hline & Sex & Right side & \multicolumn{1}{c}{ Left side } \\
\hline Inner ear & Male & $199.3 \pm 24.9$ & $205.2 \pm 26.2$ \\
& Female & $178.4 \pm 23.4$ & $181.6 \pm 27.3$ \\
Cochlea & Male & $82.8 \pm 10.3$ & $80.8 \pm 10.8$ \\
& Female & $72.8 \pm 10.5$ & $71.8 \pm 11.4$ \\
Semicircular canals & Male & $53.0 \pm 12.2$ & $55.0 \pm 12.8$ \\
& Female & $48.9 \pm 11.2$ & $50.0 \pm 12.9$ \\
Vestibule & Male & $63.8 \pm 9.0$ & $65.8 \pm 10.7$ \\
& Female & $55.4 \pm 8.1$ & $57.7 \pm 7.4$ \\
Number of inner ears & 203 & & \\
Number of patients & 118 & & \\
Number of men/women & $63 / 55$ & & \\
Mean age in years (range) & $49.4 \pm 20.4(5-87)$ & &
\end{tabular}


With regard to the different clinical diagnoses and their corresponding volumes, no statistically significant difference could be found of the inner ear, SCC, vestibule, and cochlea $(p>0.05$ for all clinical diagnoses and volumes; Table 2).

\section{Discussion}

The intention of our study was to evaluate the applicability of the most recent $\mathrm{CBCT}$ imaging technique for volumetric measurements of the bony inner ear. Moreover, we aimed to provide a range for further research. Inner ear shows a complex geometry, and volumes are extremely small. A semiautomated 3D reconstruction process was selected as a suitable method. Right and left inner ears were assessed successfully. No significant difference was found when comparing both sides. Thus, our results confirm the applicability of CBCT data for volumetric measurements. Male patients had a statistically significantly larger volume of the inner ear, the cochlea, and the vestibule. No gender-specific volume differences were found for the semicircular canals. None of the volumes correlates statistically significantly with age. There was no significant difference between the volumes when clinical diagnoses were compared. Diagnosis was hearing loss, tinnitus, vertigo, to exclude inner ear anomalies, suspected cholesteatoma, suspected otosclerosis, and surgical planning of cochlear implant or tympanoplasty. Our intention was to carry out the measurements in everyday routine with regard to the technical equipment. Volumetry of the inner ear is not performed clinically as a matter of routine.

It is worth pointing out that our results can only restrictedly be compared with those of other studies, as volumetric assessment of the osseous inner ear using CBCT has not been described before and no gold standard to assess the accuracy exists. Moreover, the methods of volume measurement differ from study to study, which makes comparisons more difficult.

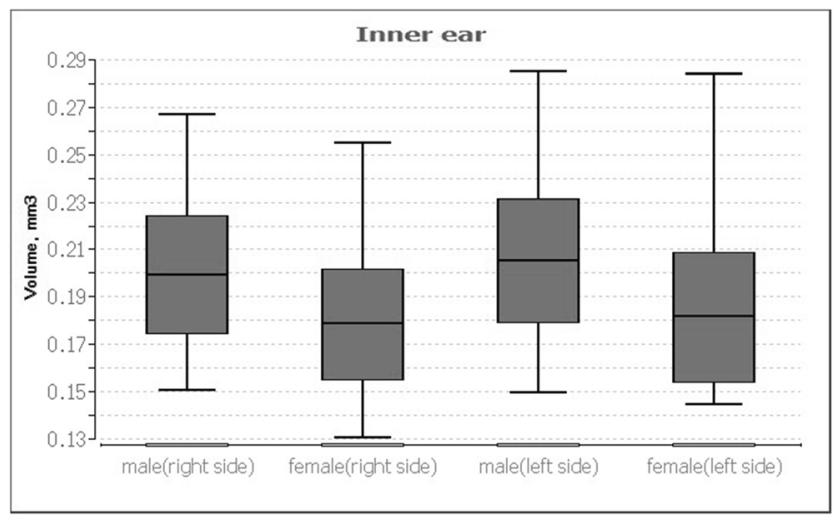

Fig. 3 Box-plots demonstrating mean male and female inner ear volumes in $\mathrm{mm}^{3}$ of the left and right side. The mean volume of the inner ear on both sides was statistically significant larger in males

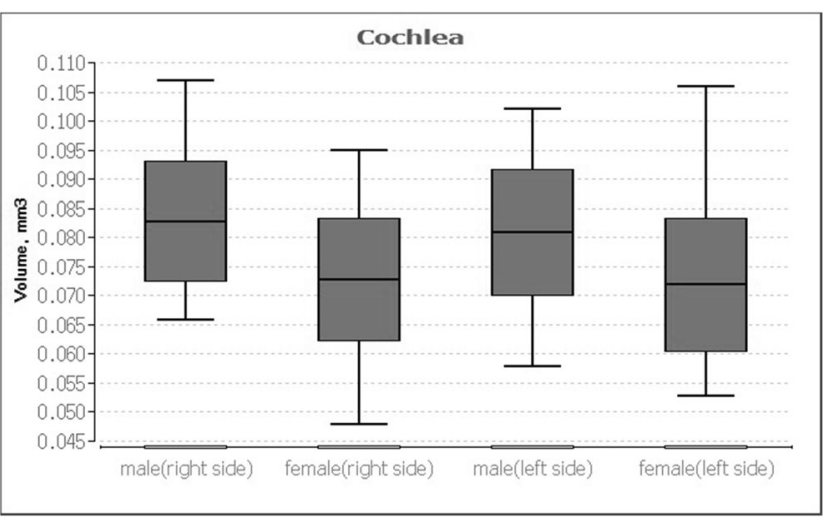

Fig. 4 Box-plots demonstrating mean male and female cochlear volumes in $\mathrm{mm}^{3}$ of the left and right side. The mean volume on both sides was statistically significant larger in males

Using MRI not all bony structures are displayed in detail [14]. If CT is used, a smaller reconstruction increment comes at the cost of a higher radiation dose $[4,16]$. CBCT provides a powerful diagnostic tool for temporal bone disorders $[7,9]$ with a high spatial resolution and three-dimensional visualization of high-contrast structures $[16,17]$. A slice thickness of up to $0.125 \mathrm{~mm}$ is possible at an acceptable low radiation dose [18], especially in limited FOV [19]. Previous studies of volumetric measurements by various researchers using CBCT included ablation volumes of the lung, pharynx, respiratory tract, skin tumors, and bones as well as the orbit and optical canal [20-23]. To assess the volume of various human organs, GE Advantage Workstation has been used before by many authors [24-26].

Our results are in the range of previous findings (Table 3). In previous studies, the mean inner ear volume ranged from 182 to $228 \mathrm{~mm}^{3}$ (median: $196 \mathrm{~mm}^{3}$ ). In our patient cohort, the mean inner ear volume amounted to $191.6 \mathrm{~mm}^{3}$. In 2001, Buckingham and Valvassori [12] measured the amount of water displaced by submerging a plastic cast. A methodological problem was a certain shrinkage artifact in the preparation

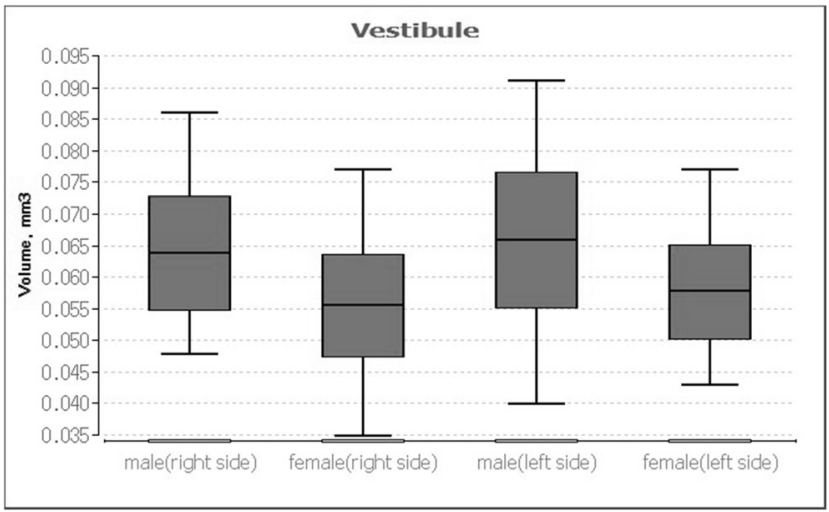

Fig. 5 Box-plots demonstrating mean male and female volumes of vestibule in $\mathrm{mm}^{3}$ of the left and right side. The mean volume on both sides was statistically significant larger in males 


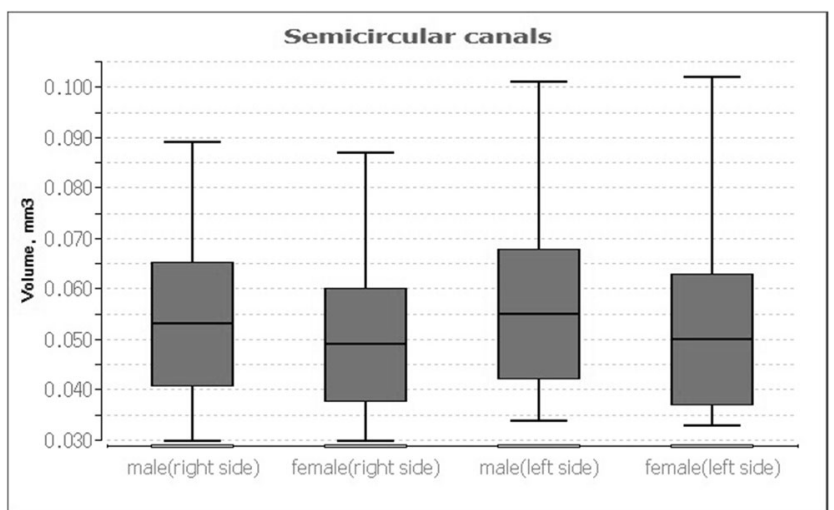

Fig. 6 Box-plots demonstrating the nonsignificant difference between males and females in the volume of the SCC on the left and right side

process. Teixido et al. [4] calculated a mean volume of $221.47 \mathrm{~mm}^{3}$. Their CT-derived inner ear volumetric measurements could not find a difference in the volume of the labyrinth between males and females, using the Amira software. Melhem et al. [14] measured a mean volume of $227.8 \mathrm{~mm}^{3}$, and no age or sex-related differences in the inner ear volumes were found. Possible age-dependent alterations of the SCC were not calculated, and they did not mention cochlear, SCC, and vestibule volumes separately. Gürkov and Berman [10] aimed to volumetrically quantify endolymph and perilymph spaces of the inner ear by using MRI sequences in only 16 patients. Their measurements are coherent with the range of ours. Kendi et al. [11] were able to measure very similar volumes of the inner ear and cochlea in only 29 healthy volunteers, despite a different methodology using MRI and a different workstation (ViStar Workstation). Igarashi et al. [27] reported the inner ear volume by manually drawing the borders of the inner ear structures to be $204.5 \mathrm{~mm}^{3}$ and the cochlear volume to be $83.6 \mathrm{~mm}^{3}$. Histological slides and a microcomputer digitizing tablet were used (Table 3).

Our results are in line with other publications in that the inner ear volume does not correlate with the patient's age. Adult size is reached at birth [14]. Age-independent bone remodeling processes that could influence the measured bony inner ear volume were reported [28]. Many other authors not only examined a smaller number of patients with different clinical pictures but also did not look at the parts of the osseous inner ear and the different disease entities separately. Enlarged fluid volumes are associated with vertigo, tinnitus, and hearing loss [29]. According to findings of Jerin et al., short-term and medium-term symptom severity fluctuations did not involve variations of the hydroptic ear [30]. Other authors report that hydrops volume was independent from vestibular symptom severity [31]. This could be an explanation of our results as we could not find different bony volumes between audiovestibular symptoms. Pathophysiological mechanisms remain unclear as we only carried out a quantitative analysis of the volume. Accurate analysis using symptom diaries or complete disease courses could reveal a stronger association of volumes and related pathologies. The final diagnosis of the patients after completion of all clinical examinations was not yet known.

Through early recognition of patients with congenital hearing loss who show a normal configuration of the inner ear but an abnormal volume, CBCT imaging might influence the severity and progression of hearing loss or balance disorders [14]. Moreover, local drug application within the inner ear is common in otolaryngology practice. Measuring the inner ear volume to estimate the concentration of transtympanic administered gentamicin for Menière disease might prevent from total deafness in patients due to too high gentamicin doses [4].

The present study has the following limitations. The study population was very heterogeneous, and their reasons to undergo CBCT might have influenced the volume. No longitudinal analysis was performed. The distinction between the temporal bone and the liquid-filled inner ear succeeded. Nevertheless, recognition of tissue borders using a threshold is fraught with possible bias.

We also arrived at the conclusion that our results should be further evaluated in homogeneous patient population.

Table 2 Comparison of the mean bony inner ear volumes between clinical diagnoses using one-way analysis of variance and Scheffe post hoc test

\begin{tabular}{llllll}
\hline Clinical diagnosis & Number of patients & Inner ear volume $\left(\mathrm{mm}^{3}\right)$ & Cochlea volume $\left(\mathrm{mm}^{3}\right)$ & SCC volume $\left(\mathrm{mm}^{3}\right)$ & Vestibule volume $\left(\mathrm{mm}^{3}\right)$ \\
\hline Hearing loss & 20 & $186.1 \pm 20.1$ & $74.4 \pm 12.9$ & $51.0 \pm 8$ & $60.3 \pm 8.1$ \\
Vertigo & 6 & $194.8 \pm 8.4$ & $78.5 \pm 6.1$ & $51.2 \pm 10.4$ & $66.4 \pm 8.7$ \\
Tinnitus & 3 & $163.3 \pm 19.8$ & $71.3 \pm 5.8$ & $38.7 \pm 6.8$ & $53.3 \pm 9.5$ \\
Suspected cholesteatoma & 23 & $197.2 \pm 32$ & $79.1 \pm 12.2$ & $54.2 \pm 14.4$ & $63 \pm 10.1$ \\
Suspected otosclerosis & 4 & $184.5 \pm 39.2$ & $76.1 \pm 10.9$ & $46.3 \pm 14.8$ & $57.5 \pm 14.2$ \\
Surgical planning & 57 & $192.8 \pm 26.1$ & $77.8 \pm 11.1$ & $51.4 \pm 10.8$ & $60.5 \pm 9.7$ \\
Exclude inner ear anomalies & 5 & $205 \pm 42.1$ & $80.1 \pm 12$ & $57.8 \pm 24.5$ & $65.4 \pm 8.1$
\end{tabular}

There was no significant difference $\left(p>0.05\right.$ for all clinical diagnoses and volumes). Data are mean \pm standard deviation in mm ${ }^{3} . S C C$, semicircular canals 
Table 3 Comparison of our findings with other studies

\begin{tabular}{|c|c|c|c|c|c|}
\hline Study Source & Modality & Inner ear volume $\left(\mathrm{mm}^{3}\right)$ & Cochlea volume $\left(\mathrm{mm}^{3}\right)$ & SCC volume $\left(\mathrm{mm}^{3}\right)$ & Vestibule volume $\left(\mathrm{mm}^{3}\right)$ \\
\hline \multirow[t]{2}{*}{ Our study (2018) } & \multirow[t]{2}{*}{$\mathrm{CBCT}$} & 189.5 (right side) & 78.1 (right side) & 51.5 (left side) & 35 (right side) \\
\hline & & 193.7 (left side) & 76.4 (left side) & 52.6 (left side) & 40 (left side) \\
\hline Gürkov et al. (2015) & MRI & 182 & 68 & 34 & 80 \\
\hline Teixido et al. (2015) & $\mathrm{CT}$ & 221.47 & - & - & - \\
\hline Igarashi et al. (1986) & Histological slices & 204.5 & 83.6 & 120.9 & \\
\hline Melhem et al. (1998) & MRI & 227.8 & - & - & - \\
\hline $\begin{array}{l}\text { Buckingham/Valvassori } \\
\text { (2001) }\end{array}$ & Plastic cast & 192.5 & 83.8 & 54.2 & 40 \\
\hline Pelliccia et al. (2014) & $\mathrm{CT}$ & - & 185.4 & - & - \\
\hline \multirow[t]{2}{*}{ Kendi et al. (2005) } & \multirow[t]{2}{*}{ MRI } & 195.7 (right side) & 84.5 (right side) & 113.82 (right side) & \\
\hline & & 196.3 (left side) & 82.9 (left side) & 112.9 (left side) & \\
\hline
\end{tabular}

Data are mean in $\mathrm{mm}^{3} . C T$, computed tomography; $M R I$, magnetic resonance imaging; $S C C$, semicircular canals

\section{Conclusions}

In conclusion, three-dimensional reconstruction and assessment of the volumetric measurements of the inner ear can be obtained using high-resolution CBCT imaging. There were no significant differences between the bony inner ear volumes and the clinical diagnosis. Our volumetric study provides an important basis for further evaluation of pathologies.

Authors' Contributions All authors contributed to the study conception and design. Material preparation, data collection, and analysis were performed by Jan Hiller, Marc Harth, and Nagy Naguib Naeem Naguib. The first draft of the manuscript was written by Jan Hiller, and all authors commented on previous versions of the manuscript. All authors read and approved the final manuscript.

Funding Open Access funding enabled and organized by Projekt DEAL.

\section{Compliance with Ethical Standards}

Conflict of Interest The authors declare that they have no conflict of interest.

Ethical Approval All procedures performed in studies involving human participants were in accordance with the ethical standards of the institutional and/or national research committee and with the 1964 Helsinki declaration and its later amendments or comparable ethical standards.

Informed Consent Informed consent was obtained from all individual participants included in the study.

Open Access This article is licensed under a Creative Commons Attribution 4.0 International License, which permits use, sharing, adaptation, distribution and reproduction in any medium or format, as long as you give appropriate credit to the original author(s) and the source, provide a link to the Creative Commons licence, and indicate if changes were made. The images or other third party material in this article are included in the article's Creative Commons licence, unless indicated otherwise in a credit line to the material. If material is not included in the article's Creative Commons licence and your intended use is not permitted by statutory regulation or exceeds the permitted use, you will need to obtain permission directly from the copyright holder. To view a copy of this licence, visit http://creativecommons.org/licenses/by/4.0/.

\section{References}

1. Attyé A, Eliezer M, Galloux A, Pietras J, Tropres I, Schmerber S, et al. Endolymphatic hydrops imaging: differential diagnosis in patients with Meniere disease symptoms. Diagn Interv Imaging. 2017;98:699-706.

2. Naganawa S, Kawai H, Sone M, Ikeda M. Ratio of vestibular endolymph in patients with isolated lateral semicircular canal dysplasia. Magn Reson Med Sci. 2015;14:203-10.

3. Purcell D, Johnson J, Fischbein N, Lalwani AK. Establishment of normative cochlear and vestibular measurements to aid in the diagnosis of inner ear malformations. Otolaryngol Head Neck Surg. 2003;128:78-87.

4. Teixido MT, Kirkilas G, Seymour P, Sem K, Iaia A, Sabra O, et al. Normative inner ear volumetric measurements. J Craniofac Surg. 2015;26:251-4.

5. Mozzo P, Procacci C, Tacconi A, Martini PT, Andreis IA. A new volumetric $\mathrm{CT}$ machine for dental imaging based on the cone-beam technique: preliminary results. Eur Radiol. 1998;8:1558-64.

6. Schulze R. Dentale digitale Volumentomographie - Technik und Anwendung. wissen kompakt. 2014;8:13-28.

7. Kösling S. Moderne Schläfenbeinbildgebung. HNO. 2017;65:46271.

8. Hofmann E, Schmid M, Lell M, Hirschfelder U. Cone beam computed tomography and low-dose multislice computed tomography in orthodontics and dentistry: a comparative evaluation on image quality and radiation exposure. J Orofac Orthop. 2014;75:384-98.

9. Dalchow CV, Weber AL, Yanagihara N, Bien S, Werner JA. Digital volume tomography: radiologic examinations of the temporal bone. AJR Am J Roentgenol. 2006;186:416-23.

10. Gurkov R, Berman A, Dietrich O, Flatz W, Jerin C, Krause E, et al. MR volumetric assessment of endolymphatic hydrops. Eur Radiol. 2015;25:585-95.

11. Kendi TK, Arikan OK, Koc C. Volume of components of labyrinth: magnetic resonance imaging study. Otol Neurotol. 2005;26:77881 . 
12. Buckingham RA, Valvassori GE. Inner ear fluid volumes and the resolving power of magnetic resonance imaging: can it differentiate endolymphatic structures? Ann Otol Rhinol Laryngol. 2001;110: 113-7.

13. Igarashi M, Ohashi K, Ishii M. Morphometric comparison of endolymphatic and perilymphatic spaces in human temporal bones. Acta Otolaryngol. 1986;101:161-4.

14. Elias R. Melhem, Huzeifa Shakir, Sivi Bakthavachalam, C. Bruce MacDonald, John Gira, Shelton D. Caruthers, and Hernan Jara. Inner ear volumetric measurements using high-resolution 3D T2weighted fast spin-echo MR imaging: initial experience in healthy subjects.

15. Komori M, Miuchi S, Hyodo J, Kobayashi T, Hyodo M. The gray scale value of ear tissues undergoing volume-rendering high-resolution cone-beam computed tomography. Auris Nasus Larynx 2018.

16. Dalchow CV, Knecht R, Grzyska U, Muenscher A. Radiographic examination of patients with dehiscence of semicircular canals with digital volume tomography. Eur Arch Otorhinolaryngol. 2013;270: 511-9.

17. Miracle AC, Mukherji SK. Conebeam CT of the head and neck, part 2: clinical applications. AJNR Am J Neuroradiol. 2009;30: 1285-92.

18. Schulze D, Heiland M, Thurmann H, Adam G. Radiation exposure during midfacial imaging using 4- and 16-slice computed tomography, cone beam computed tomography systems and conventional radiography. Dentomaxillofac Radiol. 2004;33:83-6.

19. Szabo BT, Aksoy S, Repassy G, Csomo K, Dobo-Nagy C, Orhan $\mathrm{K}$. Comparison of hand and semiautomatic tracing methods for creating maxillofacial artificial organs using sequences of computed tomography (CT) and cone beam computed tomography (CBCT) images. The International Journal of Artificial Organs. 2017;40:307-12

20. Ierardi AM, Petrillo M, Xhepa G, Laganà D, Piacentino F, Floridi $\mathrm{C}$, et al. Cone beam computed tomography images fusion in predicting lung ablation volumes: a feasibility study. Acta Radiol. 2016;57:188-96.

21. Shahidi S, Momeni Danaie S, Omidi M. Comparison of the pharyngeal airway volume between non-syndromic unilateral cleft palate and normal individuals using cone beam computed tomography. J Dent (Shiraz). 2016;17:268-75.
22. Brodin NP, Tang J, Skalina K, Quinn TJ, Basu I, Guha C, et al. Semi-automatic cone beam CT segmentation of in vivo pre-clinical subcutaneous tumours provides an efficient non-invasive alternative for tumour volume measurements. Br J Radiol. 2015;88: 20140776.

23. Friedrich RE, Bruhn M, Lohse C. Cone-beam computed tomography of the orbit and optic canal volumes. J Craniomaxillofac Surg. 2016;44:1342-9.

24. Bogot NR, Kazerooni EA, Kelly AM, Quint LE, Desjardins B, Nan B. Interobserver and intraobserver variability in the assessment of pulmonary nodule size on CT using film and computer display methods. Acad Radiol. 2005;12:948-56. https://doi.org/10.1016/j. acra.2005.04.009.

25. Estes JL, Tsiouris AJ, Christos PJ, Lelli GJ. Three-dimensional volumetric assessment of the nasolacrimal duct in patients with obstruction. Ophthalmic Plast Reconstr Surg. 2015;31:211-4.

26. Caldwell DP, Pulfer KA, Jaggi GR, Knuteson HL, Fine JP, Pozniak MA. Aortic aneurysm volume calculation: effect of operator experience. Abdom Imaging. 2005;30:259-62.

27. Igarashi $\mathrm{M}, \mathrm{O}-\mathrm{Uchi} \mathrm{T}$, Isago $\mathrm{H}$, Wright $\mathrm{WK}$. Utricular and saccular volumetry in human temporal bones. Acta Otolaryngol. 1983;95: 75-80.

28. Kamakura T, Nadol JB. Evidence of osteoclastic activity in the human temporal bone. Audiol Neurootol. 2017;22:218-25.

29. Hallpike CS, Cairns H. Observations on the pathology of Ménière's syndrome: (section of otology). Proc R Soc Med. 1938;31:131736.

30. Jerin C, Floerke S, Maxwell R, Gürkov R. Relationship between the extent of endolymphatic Hydrops and the severity and fluctuation of audiovestibular symptoms in patients with Menière's disease and MRI evidence of Hydrops. Otol Neurotol. 2018;39:e123-30.

31. Fukushima M, Kitahara T, Oya R, Akahani S, Inohara H, Naganawa $\mathrm{S}$, et al. Longitudinal up-regulation of endolymphatic hydrops in patients with Meniere's disease during medical treatment. Laryngoscope Investig Otolaryngol. 2017;2:344-50.

Publisher's Note Springer Nature remains neutral with regard to jurisdictional claims in published maps and institutional affiliations. 